\title{
Method and Mathematical Modeling of Formation of Gradually and Homogeneously Oriented Linear Polymers
}

\author{
Levan Nadareishvili (D, ${ }^{1}$ Roland Bakuradze, ${ }^{1}$ Jimsher Aneli, ${ }^{2}$ Manana Areshidze, ${ }^{1}$ \\ Ineza Pavlenishvili, ${ }^{1}$ Liana Sharashidze, ${ }^{1}$ and Giorgi Basilaia ${ }^{2}{ }^{2}$ \\ ${ }^{1}$ Vl. Chavchanidze Institute of Cybernetics of the Georgian Technical University, 5 Z. Anjaparidze St., Tbilisi 0186, Georgia \\ ${ }^{2} R$. Dvali Institute of Machine Mechanics, 10 Mindeli Str. Tbilisi 0186, Georgia \\ Correspondence should be addressed to Levan Nadareishvili; levannadar@yahoo.com
}

Received 2 April 2019; Accepted 1 October 2019; Published 25 November 2019

Academic Editor: Domenico Acierno

Copyright (c) 2019 Levan Nadareishvili et al. This is an open access article distributed under the Creative Commons Attribution License, which permits unrestricted use, distribution, and reproduction in any medium, provided the original work is properly cited.

\begin{abstract}
Previously, we developed several technical solutions for the conversion of isotropic polymers into materials of a new type-gradually oriented polymers with a gradient of physical and mechanical properties, thereby materializing for the first time the unused possibilities inherent in the polymeric nature of the substance. The scientific basis of these developments is the concept of a new structural state of linear polymers-a gradually oriented (stretched) state (GOS), the essence of which is set out briefly. An algorithm and a mathematical model for controlling the process of uniaxial, zonal stretching of linear polymers are proposed, which allow the formation of gradually or homogeneously oriented polymers (polymer composites). At the same time, specified values of quantitative parameters are ensured, in particular, the selected profile of distribution of the relative elongation (linear, sinusoidal, etc.) along the length of the gradually oriented polymer sample and the specified constant relative elongation along the length of the homogeneously oriented polymer. Mathematical expressions for calculating the conditions to obtain gradually oriented polymers with given geometric dimensions (length, width) and with a given distribution of relative elongation along the length of the resulting sample were also derived. The description of method and principles of operation of the appropriate device is given. Experimental data illustrating the possibilities of the proposed method and the efficiency of mathematical modeling are presented. The issues of creating functionally graded materials with a gradient of microstructure (gradient of degree of orientation/relative elongation) or chemical composition (volume fraction of functional fillers), respectively, based on thermoplastic polymer/copolymer or polymer composite (containing functional fillers) by method of uniaxial, zonal graded stretching are discussed.
\end{abstract}

\section{Introduction}

1.1. Background. Uniaxial orientational stretching is a widespread method of structural modification of the linear polymers [1-3]. As a result of stretching at the above glass transition temperature, the isotropic polymer goes over into the oriented state, which is characterized by a preferential location of the structural elements in the stretching direction. The material becomes anisotropic. The structural anisotropy leads to the considerable change in many physical and mechanical properties. Therefore, the study of orientation processes is closely associated to the development of polymer science and polymer industry.
There are various technical solutions for converting a polymer into an oriented state: cold or hot stretching $[4,5]$, extruding solid polymer [4-7], rapid stretching from melts or solutions $[4,8,9]$, and zonal stretching [10-12].

The polymer samples with different properties, films in particular, can be obtained by varying the degree of orientation, which depends on the following parameters: stretching temperature, stretching speed (in conditions of the same relative elongations), cooling speed of the heated polymer, and relative elongation $(\lambda)$.

If during the stretching of the polymer we provide a gradient of one of the four aforementioned parameters, for example, the gradient of relative elongation $(\lambda)$, then the 
gradient of the degree of orientation and, consequently, the gradient of physical and mechanical properties should be formed. The validity of this consideration is indicated by the relationship between the birefringence and relative elongation [13]:

$$
\Delta n=n_{1}-n_{2}=\gamma \lambda
$$

where $n_{1}$ and $n_{2}$ are the index of refraction of ordinary and extraordinary rays, respectively, $\gamma$ is the optical coefficient of deformation, and $\lambda$ is the relative elongation. From this equation, it follows that the gradient of relative elongation should cause the gradient of birefringence.

If we abstract from the foregoing, wide unused resources will be revealed that exist in the polymer nature of the substance due to the flexibility of the macromolecules and, as a result, the property of the change in the conformational set. This is the ability to create a new type of material-gradient-oriented polymers (composites) with the gradient of many physical and mechanical properties.

This, in turn, requires expansion of the concept of an oriented state and a new understanding of the structural features of oriented polymers, developing new technical solutions for orientational stretching, and determination of the appropriate quantitative regularities.

Paying a tribute to the historical justice, it should be noted that almost half a century ago Shen and Bever [14] apparently first noted the importance of investigation of gradient polymeric materials. The authors point to different ways to create a gradient. With respect to the gradient of degree and direction of orientation, the authors have limited themselves to pointing out the undeniable importance of studying this problem without giving any specific solutions for implementing this idea. This issue was put into practice for the first time by us.

We have developed various methods (stretching in inhomogeneous mechanical field [15-17], controlled extension of the stretching zone [18, 19], and controlled zonal stretching $[20,21])$ for converting isotropic polymers (polymer composites) into a gradually oriented state (GOS) and have established quantitative relationships for the process of forming a gradually oriented structure for some specific configuration of a tensile device [22-29]. The structural feature of GOS is the gradient of the preferred arrangement of structural elements (macromolecular chains and their parts, as well as supermolecular structural fragments) in the stretching direction. Quantitatively, the degree of orientation in GOS can be characterized by the orientation functions $\overline{\cos ^{2} \theta}$ and $\overline{\cos ^{4} \theta}(\theta$ is the angle between the stretching direction and the axis of a given section of the macromolecule) [30]. GOS can be also described with function $\lambda=f(L)$ where $\lambda=\Delta \ell / \ell, \lambda$ is the relative elongation, $\Delta \ell$ is the lengthening of the sector, $\ell$ is the initial sector length, and $L$ is the coordinate of the sample (length of the sample). Relative elongation varies within the $0 \leq \lambda \leq n$ range, wherein the maximum value of $n$ for currently existing thermoplastic polymers may be $8 \div 10$. It is necessary to underline that the degree of orientation is uniquely depen-

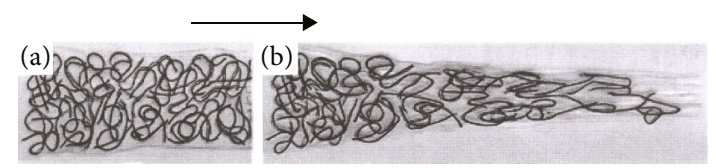

FIgURE 1: Scheme of transformation of an isotropic amorphous polymer in the process of gradient stretching (transformation of a statistical coil): (a) isotropic polymer film and (b) the same film gradually stretched. The arrow indicates the direction of propagation of the extension front.

dent on the relative elongation under the immutability of other factors of stretching mode (temperature, stretching velocity) [31-33].

The scheme we have created is shown in Figure 1. It illustrates the essence of the gradient stretching by the example of a change in the conformation of macromolecules - the transformation of a statistical coil $[34,35]$ into an isotropic sample of an amorphous polymer (Figure 1(a)) during its gradient zonal stretching.

As the degree of stretching increases in the local region of the sample, the statistical coil gradually unfolds and straightens in the direction of propagation of the stretching front (Figure 1(b)). The relative elongation $\lambda$ of the separate parts of the sample gradually increases from 0 to about 2.5 .

The optical, electrical, and magnetic properties of gradually oriented polymer systems are studied. By the example of gradient-oriented films of polyvinyl alcohol and polyethylene terephthalate, a good correlation is established between the values of relative elongation and birefringence [22-27, 36]. Investigations of electric and magnetic properties of gradually stretched polymer composite films (polyvinyl alcohol with graphite, carbon black, and nickel) show that the character of change of these properties essentially depends both on the composition of materials and their degree of elongation [37-40].

It was also shown that inhomogeneous stretching of a polyvinylidene fluoride plate leads to a gradient phase transformation $\alpha \longrightarrow \beta$ and, accordingly, to a gradient of piezoelectric and optical properties [41].

These studies indicate that gradient orientation is an effective scientific and technical approach for creating materials of a new type. We believe that these studies are only the first steps in a broad, practically unexplored, perspective scientific field, which opens up great opportunities for obtaining new type of gradient materials with different physical and mechanical properties.

Further development of this promising direction, among other things, is connected with the development of new technical solutions and the establishment of quantitative patterns of the orientational stretching process. This article proposes an algorithm and a mathematical model for managing the process of formation of gradually and homogeneously oriented polymeric materials by the uniaxial, zonal stretching method. The experimental data illustrating the possibilities of the method and the efficiency of mathematical modeling are presented. The outlooks for creating functionally graded materials by the methods of gradient stretching of the thermoplastic polymers (composites) are also discussed. 
The equidistant parallel lines are plotted on the surface of the isotropic film to record the distribution of the elongation (a)

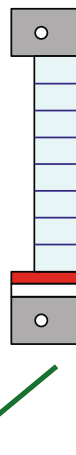

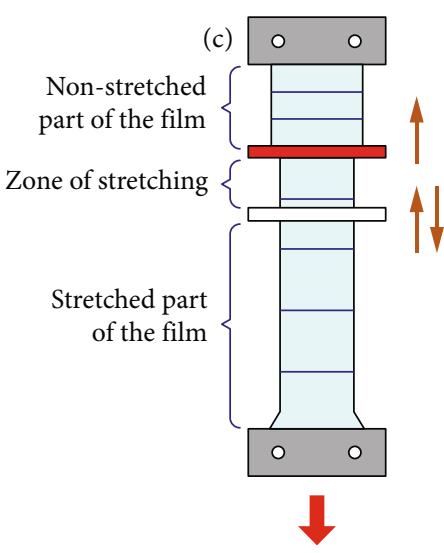

(d)

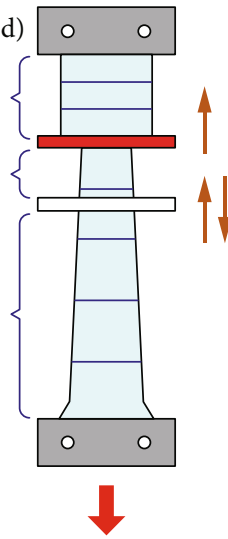

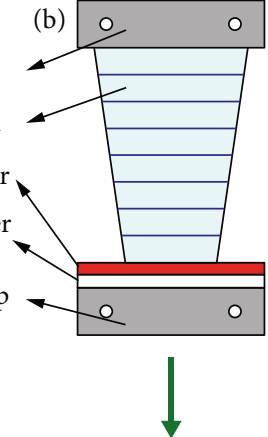

(e)

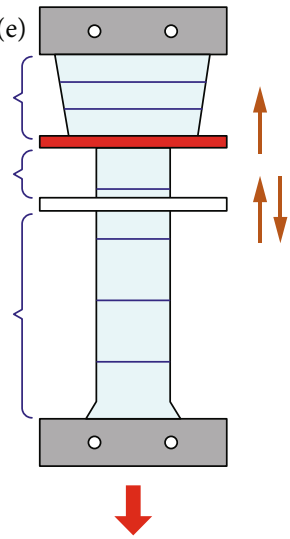

(f)

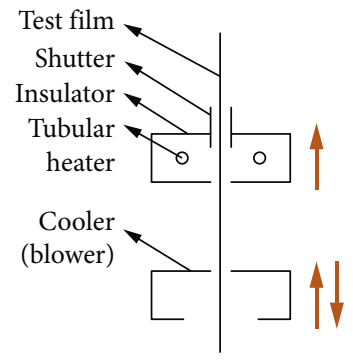

1 Direction of motion of the zonal heater

A Direction of motion

Ty of the cooler

Direction of motion of the active clamp

Figure 2: Zonal stretching scheme. Isotropic films with rectangular (a) and trapezoidal (b) forms. (c) Scheme of zonal homogeneous (nongradient) stretching of the (a) rectangular film. (d) Scheme of zonal graded stretching of the (a) rectangular film. (e) Scheme of zonal gradient stretching of the (b) trapezoidal film. (f) Mutual arrangement of the test film, zonal heater unit, and cooler (side view).

\section{Materials and Methods}

2.1. Research Materials. The method of uniaxial, zonal stretching is based on the ability of materials to have large deformations. Therefore, any thermoplastic polymers, copolymers, and composites with a polymer matrix containing inorganic (ceramics, metal), organic, or polymeric fillers can be used as test materials. The method cannot be used for structural modification of samples having a complex, volumetric shape. Therefore, the starting and received products are films, plates, rods, or fibers.

In this article, polyvinyl alcohol films were used as the model material. The films are prepared as follows: after swelling in distilled water ( 24 hours) and boiling ( 24 hours), a hot $10 \div 15 \%$ polymer solution is poured onto a horizontal glass surface and the solvent is evaporated at room temperature. Highly transparent films with a size of about $200 \times 300 \mathrm{~mm}$ and a thickness of $0.1 \div 0.3 \mathrm{~mm}$ are obtained. Samples of the desired size are cut out from an isotropic film. To register the distribution of elongation along the length of the specimen as a result of stretching, equidistant parallel lines are applied to its surface perpendicularly to the direction of the subsequent stretching. Uniaxial zonal stretching of samples (gradient or homogeneous) is carried out on a device developed by us (the description is given below) at $373 \mathrm{~K}$ and a zonal heater moving speed of $10 \mathrm{~mm} / \mathrm{min}$. The speeds of the active cooler and the active clamp are determined on the basis of the proposed model.
2.2. Uniaxial Zonal Controlled Stretching Method. The distinctive features of the method under consideration are as follows: application of an active cooler in the stretching process, adjustment of the extent of the stretching zone, and adjustment of speed/acceleration and direction of movement of the active cooler. According to [21], the zonal stretching device (ZSD) consists of three structurally independent units: a tensile testing machine or its simplified analog, an add-on module (zonal heater, active cooler, and mechanism for their movement), and a stretching process control block. On the $\mathrm{ZSD}$, it is possible to stretch polymer samples both in homogeneous (nongradient) and in gradient modes and to ensure the specified distribution of relative elongation along the length of the resulting sample.

Consider the operation of the ZSD using rectangularand trapezoidal-shaped films (Figures 2(a) and 2(b)). The test sample is fastened between fixed and active clamps. Zonal heaters and coolers (blowers) are located on both sides of the sample. A more detailed pattern of the heating element and cooler is shown in Figure 2(f). Heaters and coolers have an equidistant geometric shape relative to the test sample and are arranged perpendicularly to the stretching direction. Tubular heaters are placed in heat insulators. The heat insulators are equipped with shutters for regulating the clearance of the channels guiding the heat flow. Heat insulators and coolers are connected to electric engines (not shown). At the beginning of the stretching process, the coolers are located near the active clamps. Heaters are located between 


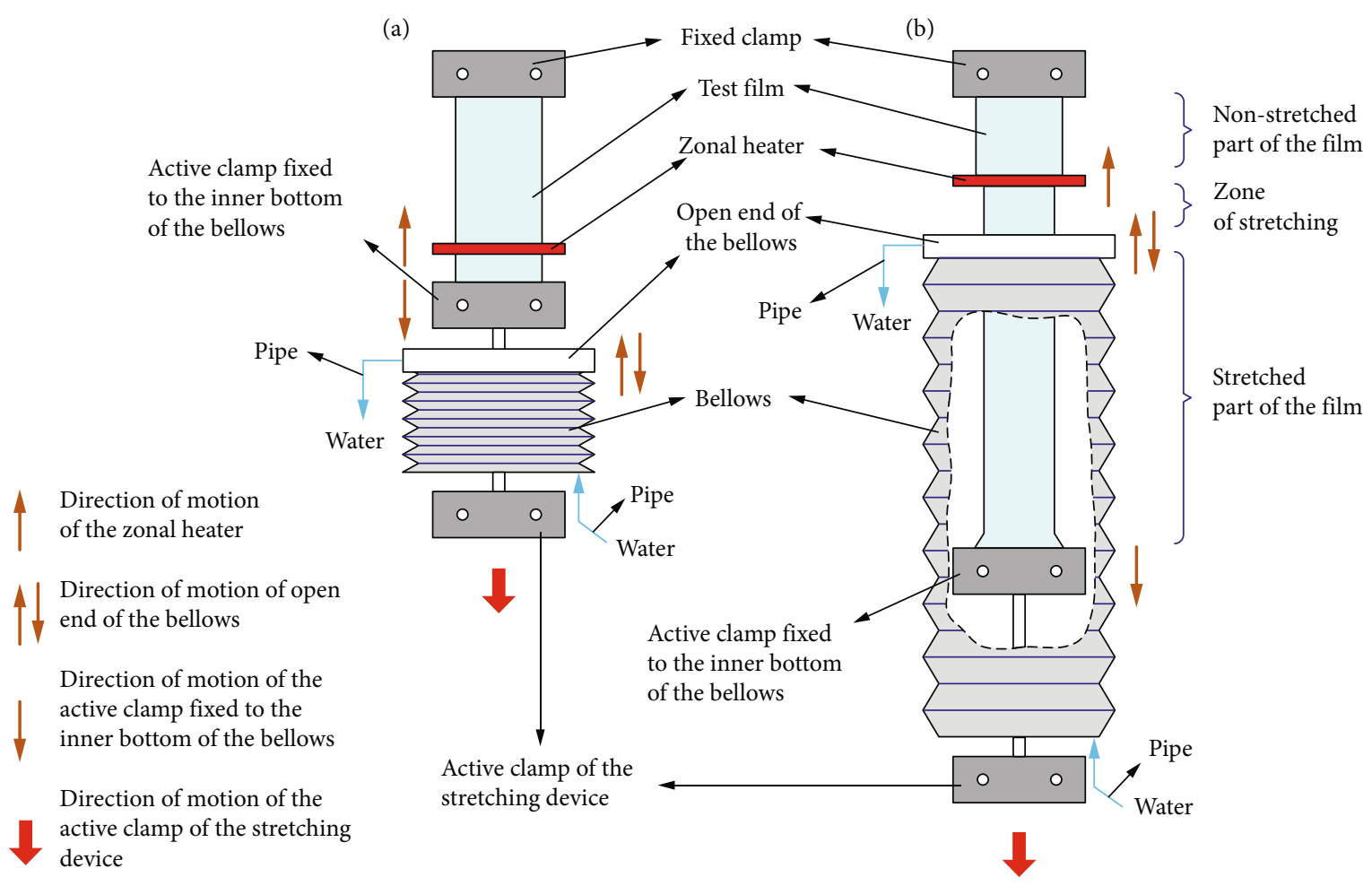

Figure 3: Scheme of using bellows with liquid for cooling the test sample during the stretching process: (a) initial state and (b) stretching process.

the coolers and the fixed clamps. The motion mode of heaters, coolers, and active clamps is controlled by the control unit (not shown).

2.3. Zonal Homogeneous Stretching. Figure 2(c) shows the scheme of zonal homogeneous (nongradient) stretching of a rectangular film (Figure 2(a)). The sample is heated in a narrow zone by zonal heaters. The yield point of polymer sample decreases in the heated zone. When active clamps are moved, a part of the sample in the heated zone will stretch. The stretching stops in the cooling zone. Consequently, elongation of the sample is strictly limited by the distance between heaters and coolers. By simultaneously moving the active clamps, heaters, and coolers, the rest of the sample will also be stretched. In the homogeneous stretching process, active clamps, heaters, and coolers move at a constant speed. The ratio of these speeds, the distance between heaters and coolers, and the height of the heaters determine the value of the local elongation (see below).

2.4. Zonal Graded Stretching. Figure 2(d) shows a zonal graded stretching scheme for the rectangular film. In this case, the heating, stretching, and cooling of the sample are carried out as described above. Under conditions of a constant height of the heaters and a constant speed of their movement, the gradient stretching is achieved by ensuring proper acceleration of the displacement of the active clamps. At the same time, simultaneous adjustment of the extent of the stretch zone (the distance between the heater and the cooler) is achieved by selecting speed (con- stant or accelerated) and direction of motion of the cooler. Stretching regime is determined on the basis of a mathematical model (see below).

In the process of zonal graded stretching, an increase of velocity of movement of the active clamp (i.e., an increase in relative elongation) leads to an increase in the transverse shrinkage of the sample. Rectangular isotropic film (Figure 2(a)) transforms into a gradually oriented trapezoidal film (Figure 2(d)). If the original film has a trapezoidal shape (Figure 2(b)), its stretching in the same regime gives a rectangular one (Figure $2(\mathrm{e})$ ). The necessary geometric dimensions of the initial trapezoid can be determined on the basis of an experiment or by the use of Poisson's ratio.

2.5. Liquid Cooling. The test sample can be cooled with liquid (water) in a vessel with a variable volume, for example, a bellows. Figure 3(a) shows the initial state of the ZSD, equipped with bellows.

Between the fixed and active clamps, the test film is fastened. The active clamp is fixed to the inner bottom of the bellows, and the bellows is in turn attached to the active clamp of the stretching device. The bellows is equipped with pipes for water inlet and outlet. The upper open end of the bellows is connected to the electric engine (not shown). In the process of stretching the sample, the bellows unfolds. With a controlled motion of the upper open end of the bellows (similar to cooler in Figure 2), the height of the stretching zone of the sample is adjusted. Stretching stops when the sample is immersed in a cooling 
liquid. Figure 3(b) shows the ZSD with a partially stretched sample.

The stretching of the sample (homogeneous or gradient mode) is realized in accordance with the algorithm considered below.

\section{Mathematical Modeling of Uniaxial Zonal Homogeneous and Graded Stretching}

The following subjects are considered below:

(i) Algorithm design for homogeneous and gradient zonal stretching

(ii) Derivation of mathematical equations for calculating the conditions of obtaining homogeneously and gradually oriented samples with a given distribution of the relative elongation

(iii) Calculation of the total elongation of an isotropic sample with a length $L$ if the predetermined distribution of the relative elongation is realized along its entire length

(iv) Calculation of the initial length $L$ of the test sample, the gradient stretching of which will lead to the formation of a sample with a predetermined distribution of the relative elongation and a length $L+\Delta L$

The model is approximate. The model more accurately describes the stretching process the smaller the height of the zonal heater. The model does not consider the physical processes that occur during stretching. At the same time, it is assumed that the heat transfer is carried out only along the direction of the thickness of the sample (the heat transfer along the length of the sample is so insignificant that it can be neglected). Consequently, only the heated zone of the sample is subjected to stretching.

We introduce the following notations:

$L$ : Initial length of isotropic sample,

$\Delta L$ : Total elongation of isotropic sample as a result of stretching,

$\ell$ : Height of the heating zone in the sample $(\ell=$ const $)$,

$T$ : Duration of the stretching process,

$t$ : Moment of the sample stretching process $(t \in(0 ; T))$, $\Delta \ell(t)$ : Elongation of $\ell$ zone of the sample at moment $t$,

$\lambda(t)$ : Relative elongation of $\ell$ zone at moment $t$,

$V_{1}$ : Zonal heater moving speed at moment $t\left(V_{1}=\right.$ const $)$,

$V_{2}(t)$ : Cooler (upper open end of bellows) moving speed at moment $t$,

$V_{3}(t)$ : Active clamp moving speed at moment $t$,

$h(t)$ : Distance between zonal heater and cooler (upper open end of bellows) at moment $t$,

$s_{1}(t)$ : Distance passed by zonal heater at moment $t$,

$s_{2}(t)$ : Distance passed by cooler (upper open end of bellows) at moment $t$,

$s_{3}(t)$ : Distance passed by active clamp at moment $t$,

$\lambda$ : Relative elongation calculated with respect to the heated zone $\ell$.
We write the zonal homogeneous and graded stretching conditions in the general form. The condition of zonal homogeneous stretching is

$$
\begin{aligned}
& V_{1}=V_{2}=\text { const }, \\
& V_{3}=\text { const } .
\end{aligned}
$$

Taking into account the equality of the first two velocities, it is clear that in the process of zonal homogeneous stretching $h(t)=$ const.

From the various possible modes of zonal gradient orientation, consider the following combination of speeds of mobile components of the ZSD:

$$
\begin{aligned}
& V_{1}(t)=\text { const }, \\
& V_{2}(t)=\text { const }, \\
& V_{3}(t) \neq \text { const. }
\end{aligned}
$$

This combination leads to a rectilinear distribution of the relative elongation. In all cases of graded stretching, $h(t) \neq$ const.

The zonal heater passes $\ell$ distance in time $t$. During the same time, as a result of moving of the active clamp, the heated $\ell$ zone of the sample is lengthened by $s_{3}(t)=\Delta \ell(t)$. At time $t$, the relative elongation of the heated zone is

$$
\lambda(t)=\frac{\Delta \ell(t)}{\ell}
$$

The relative elongation can also be written as the ratio of speeds of movement of the active clamp and the zonal heater:

$$
\lambda(t)=\frac{V_{3}(t)}{V_{1}}
$$

At time $t$, the length of the elongated segment of the sample is $\Delta \ell(t)+\ell$ and it will be located between the heater and the cooler.

At any stage of the stretching process between the heater and the cooler, it is necessary to provide a distance equal to the length of the current elongated part of the sample:

$$
h(t)=\Delta \ell(t)+\ell=\ell(\lambda(t)+1) .
$$

In the stretching process, the set value of $h(t)$ is maintained by ensuring the appropriate speed of cooler. The cooler (upper open end of bellows) moving speed $V_{2}(t)$ is calculated as follows.

At any point in the stretching process, $h$ can be represented as the difference in distances traveled by the heater and the cooler:

$$
h(t)=s_{1}(t)-s_{2}(t)
$$

Taking into account (6), we have

$$
s_{1}(t)-s_{2}(t)=\ell(\lambda(t)+1) .
$$




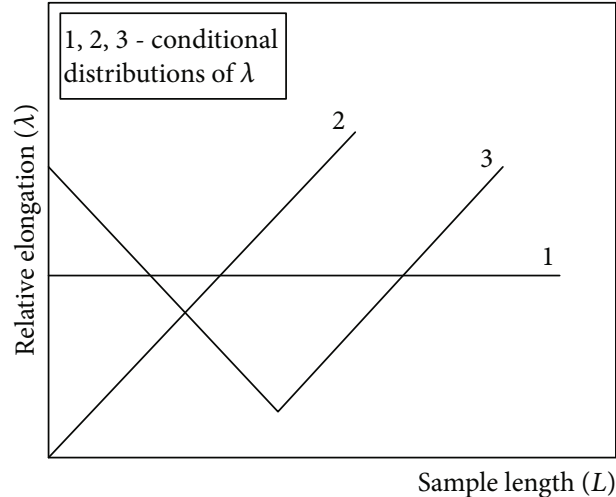

(a)

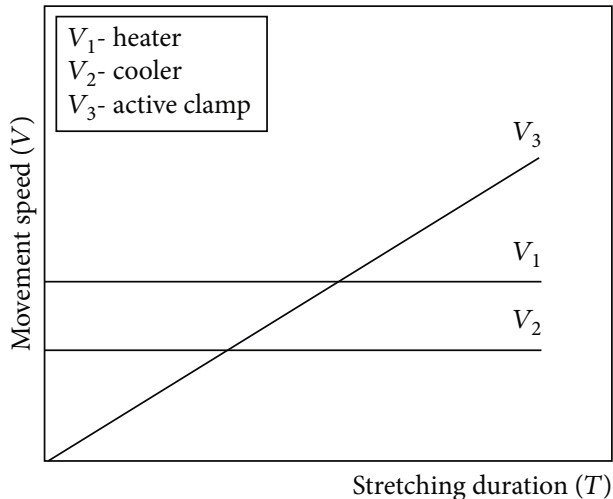

(c)

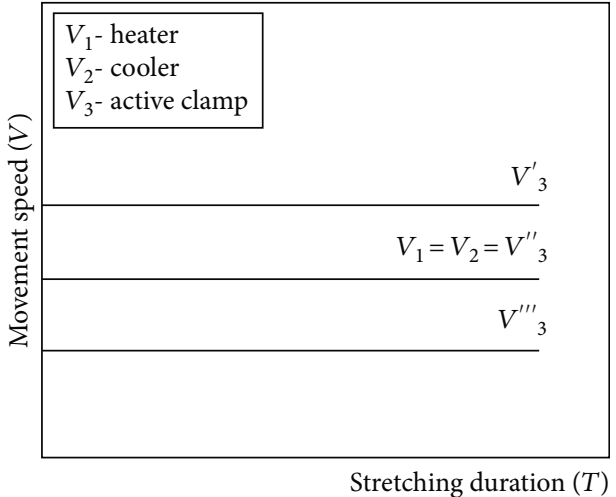

(b)

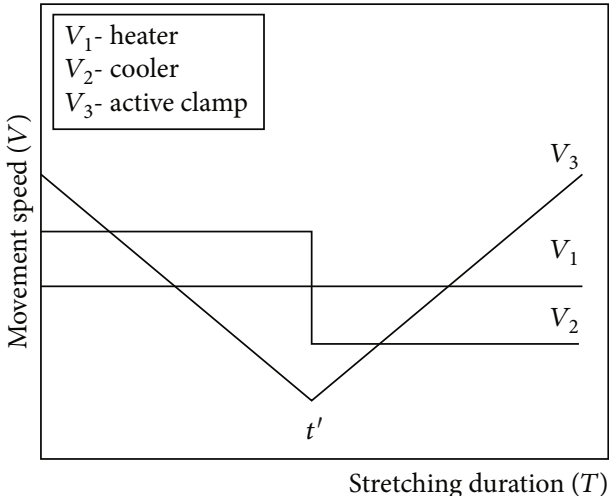

(d)

FIGURE 4: Creation of various distributions of relative elongation by appropriate combinations of motion speeds of zonal heater, active cooler, and active clamp. (a) Three cases of different possible conditional distributions of relative elongation $\lambda$ that must be created along the length $L$ of the isotropic sample. (b-d) Dependences of motion speeds $(V)$ of the heater, active cooler, and active clamp on the duration of the stretching process $(T)$, which realize the distribution of relative elongations along the length $L$ of the isotropic sample corresponding to curves 1,2 , and 3 in (a).

Since $V_{1}$ is constant, we can write

$$
V_{1} \cdot t-s_{2}(t)=\ell(\lambda(t)+1) \text {. }
$$

We differentiate (9) with respect to $t$. The speed of the cooler will be written in the following form:

$$
V_{2}(t)=V_{1}-\frac{d \lambda(t)}{d t} \cdot \ell
$$

To illustrate a zonal stretching process, let us consider several examples.

Assume it only needs to carry out a homogeneous or gradient stretching of an isotropic sample. Figure 4(a) presents the graphs of function $\lambda=f(L)$-three cases of different possible conditional distributions of relative elongation $\lambda$ that must be created along $L$ length of the test sample. The first case is realized as a result of homogeneous (nongradient) stretching, cases 2 and 3 as a result of gradient stretching.

Heater moving with speed $V_{1}=$ const, the working length $L$ of an isotropic sample passes in time $T$. Figures 4(b)-4(d) show graphs of the functions $V=f(T)$, the conditional distributions of speeds of the heater, cooler, and active clamp during the stretching process, which provide given elongations at given points of isotropic sample, thus realizing the conditions specified in Figure 4(a).

Consider zonal homogeneous stretching (Figure 4(a), curve 1, and Figure 4(b)). The value of relative elongation $\lambda$ is selected. From (5), the speed of the active clamp $V_{3}$ is calculated. This conditionally can be $V_{3}{ }^{\prime}, V_{3}{ }^{\prime \prime}$, or $V_{3}{ }^{\prime \prime \prime}$. Taking into account the chosen value of $\lambda$ from (7), we determine $h(0)$.

In the starting position, the distance between the heater and the cooler is zero despite the stretching regime (homogeneous or gradient). We turn on the heater. Upon reaching the desired temperature, all the moving components of the stretching device (heater, cooler, and active clamp) are simultaneously activated. At first, the cooler moves in the stretching direction with a speed of the active clamp $V_{2}=V_{3}\left(V_{3}{ }^{\prime}, V_{3}{ }^{\prime \prime}\right.$, or $\left.V_{3}{ }^{\prime \prime \prime}\right)$. As soon as the distance between the heater and the cooler reaches the calculated value $h$, the cooler changes the direction of motion and will move in the opposite direction, following the heater with a speed of $V_{2}=V_{1}$. 
The zonal gradient stretching (Figure 4(a), curve 2, and Figure $4(\mathrm{c})$ ) is carried out by the following scheme. $\lambda(t)$ is selected. From (6) and (10), we determine correspondingly $V_{3}(t)$ and $V_{2}(t)$. We turn on the heater. When the desired temperature is reached, the heater, the cooler, and the active clamp are simultaneously activated. The cooler moves in the direction of motion of the heater with speed $V_{2}=$ const and $V_{2}<V_{1}$, so that the distance between it and the heater continuously increases and at any time is equal to $h(t)(7)$.

The zonal gradient stretching (Figure 4(a), curve 3, and Figure 4(d)) is carried out by the following scheme. $\lambda(t)$ is selected. From (5) and (10), we determine correspondingly $V_{3}(t)$ and $V_{2}(t)$. In the case under consideration, $\lambda(0)>0$. With this in mind, $h(0)$ is determined from (5). We turn on the heater. After achieving the required temperature, the heater, cooler, and active clamp will move at the same time. At first, the cooler will move in the stretching direction and with the speed of movement of the active clamp $V_{2}(t)=V_{3}(t)$. When the distance between the heater and cooler will be equated to $h(0)$, the cooler will change the direction of travel and will move in the direction of the heater movement at speed $V_{2}(t)=$ const and $V_{2}(t)>V_{1}$. From moment $t^{\prime}$, the speed of the cooler will become $V_{2}(t)=$ const and $V_{2}(t)<V_{1} . V_{2}(t)$ will be determined from (10).

We have considered a combination of speeds of the mobile components which provides a rectilinear distribution of the relative elongation. In order to implement the curvilinear distribution of the relative elongation, the following combination of speeds can be applied:

$$
\begin{aligned}
& V_{1}(t)=\text { const }, \\
& V_{2}(t) \neq \text { const }, \\
& V_{3}(t) \neq \text { const. }
\end{aligned}
$$

We now turn to consider the next subject.

We calculate the total elongation of an isotropic sample with a length $L$ if the relative elongation $\lambda(x)$, $x \in[0, L]$, is realized along its entire length $\lambda(x) \geq 0$ and it is continuous throughout the area of the definition. The initial length $L$ of the sample is divided into $n$ equal parts. The length of each part is denoted by $\Delta x_{i}$ and the elongation of this part by $\Delta L_{i}$.

On the segment $\Delta x_{i}$ any point $\xi_{i}$ is selected. At $\xi_{i}$, the relative elongation is

$$
\begin{gathered}
\lambda\left(\xi_{i}\right)=\frac{\Delta L_{i}}{\Delta x_{i}}, \\
\Delta L_{i}=\lambda\left(\xi_{i}\right) \Delta x_{i} .
\end{gathered}
$$

Sum both sides of equality (13) from 1 to $n$ :

$$
\sum_{i=1}^{n} \Delta L_{i}=\sum_{i=1}^{n} \lambda\left(\xi_{i}\right) \Delta x_{i}
$$

We pass to the limit on both sides of equality (14) when $\Delta x_{i} \longrightarrow 0$,

$$
\lim _{\Delta x_{i} \rightarrow 0} \sum_{i=1}^{n} \Delta L_{i}=\lim _{\Delta x_{i} \rightarrow 0} \sum_{i=1}^{n} \lambda\left(\xi_{i}\right) \Delta x_{i} .
$$

From (15), it follows that the elongation of the sample with an initial length $L$ equal to

$$
\Delta L(x)=\int_{0}^{L} \lambda(x) d x, \quad x \in[0 ; L] .
$$

Consider the last subject.

Now we calculate the initial length $L$ of the test sample, the gradient stretching of which will lead to the formation of a sample with a predetermined distribution of the relative elongation and a length $L+\Delta L$.

The relative elongation along the entire length of the sample is $\lambda(x)$, where $x$ is the designation of the length of the segment $[0 ; x]$. The definition area of the function $\lambda(x)$ is the interval $[0 ; L+\Delta L]$. At the same time, $\lambda(x) \geq$ 0 and it is continuous throughout the area of the definition.

Segment $[0 ; L+\Delta L]$ is divided into $n$ equal parts. The length of each part is denoted by $\Delta x_{i}$ and the elongation of each part by $\Delta L_{i}$. On segment $\Delta x_{i}$, any point $\xi_{i}$ is selected. At $\xi_{i}$, the relative elongation is

$$
\lambda\left(\xi_{i}\right)=\frac{\Delta L_{i}}{\Delta x_{i}-\Delta L_{i}} .
$$

From (17), we get

$$
\Delta L_{i}=\frac{\lambda\left(\xi_{i}\right)}{1+\lambda\left(\xi_{i}\right)} \Delta x_{i} .
$$

Sum both sides of equality (18) from 0 to $k(k \leq n)$ :

$$
\sum_{i=1}^{n} \Delta L_{i}=\sum_{i=1}^{n} \frac{\lambda\left(\xi_{i}\right)}{1+\lambda\left(\xi_{i}\right)} \Delta x_{i}
$$

According to the clause, $\lambda(x)$ function is positive and continuous throughout the area of the definition. On both sides of the equality, we pass to the limit, when $\Delta x_{i} \longrightarrow 0$,

$$
\lim _{\Delta x_{i} \rightarrow 0} \sum_{i=1}^{n} \Delta L_{i}=\lim _{\Delta x_{i} \rightarrow 0} \sum_{i=1}^{n} \frac{\lambda\left(\xi_{i}\right)}{1+\lambda\left(\xi_{i}\right)} \Delta x_{i} .
$$

From (20), it follows that the elongation of the sample on the segment $[0 ; x]$ will be

$$
\Delta L(x)=\int_{0}^{x} \frac{\lambda(x)}{1+\lambda(x)} d x, \quad x \in[0 ; L+\Delta L] .
$$




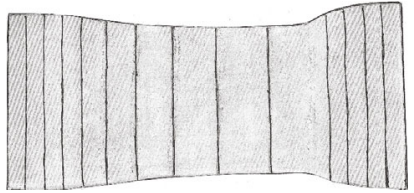

(a)

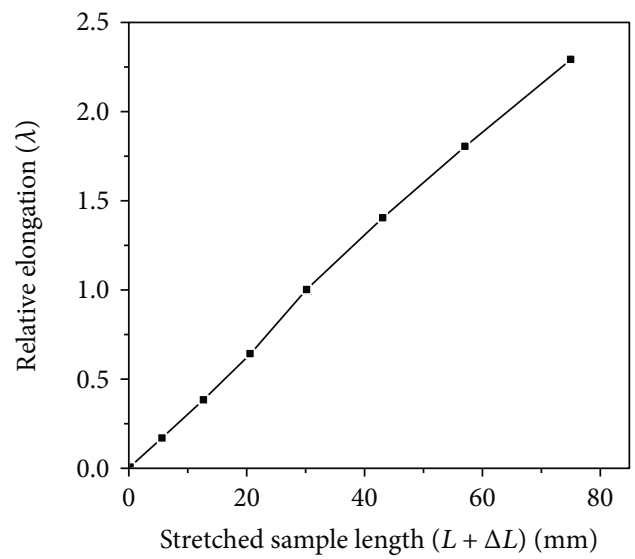

(c)

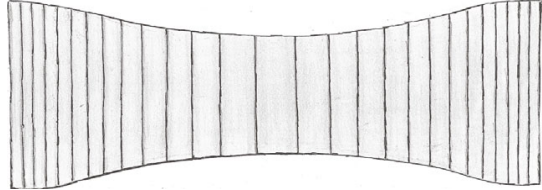

(b)

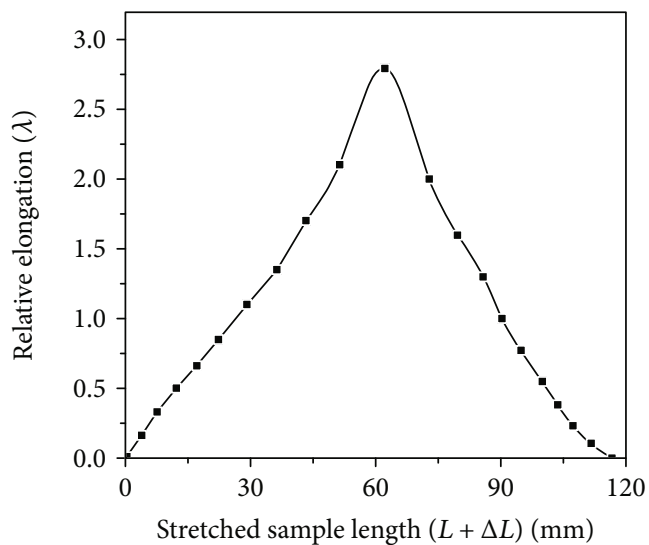

(d)

FIGURE 5: Gradually stretched polyvinyl alcohol films with different distribution of relative elongation: (a) linear and (b) sinusoidal. (c, d) Dependence of relative elongation $\lambda$ on the length of gradually oriented films $(L+\Delta L)$ presented, respectively, in (a) and (b).

From (21), we obtain that in a sample of length $L+\Delta L$ the length of the elongated part is

$$
\Delta L=\int_{0}^{L+\Delta L} \frac{\lambda(x)}{1+\lambda(x)} d x .
$$

It is clear that the length of an isotropic sample is equal to the difference between the length of the elongated sample and the total elongation:

$$
\begin{aligned}
L & =(L+\Delta L)-\int_{0}^{L+\Delta L} \frac{\lambda(x)}{1+\lambda(x)} d x \\
& =\int_{0}^{L+\Delta L} d x-\int_{0}^{L+\Delta L} \frac{\lambda(x)}{1+\lambda(x)} d x \\
& =\int_{0}^{L+\Delta L}\left(1-\frac{\lambda(x)}{1+\lambda(x)}\right) d x .
\end{aligned}
$$

That is

$$
L=\int_{0}^{L+\Delta L} \frac{d x}{1+\lambda(x)} .
$$

\section{Zonal Graded Stretching Method and Functionally Graded Materials}

The method of zonal graded stretching makes it possible to realize the selected distribution of the relative elongation $\lambda$.

Figures 5(a) and 5(b) show gradually oriented polyvinyl alcohol films obtained from rectangular samples. A sample (Figure 5(a)) was obtained by combining the speeds of mobile nodes (heater, cooler, and active clamp), the relative conditional values of which are shown in Figure 4(c) and the sample (Figure 5(b)) by the combination of speeds shown in Figure 4(d). In the second case, the stretching was first carried out by a combination of speeds represented to the right of $t^{\prime}$ on the $T$ axis and then by a combination corresponding to the time interval of stretching $0 \div t^{\prime}$.

Figures 5(c) and 5(d) show the distribution of the relative elongation $\lambda$ along the length $(L+\Delta L)$ of gradually oriented films presented in Figures 5(a) and 5(b). The total elongation of the sample with an initial length of $35 \mathrm{~mm}$ is $117 \%$, and the distribution of the relative elongation along the length of the film is linear and varies in the range $\lambda=0 \div 2.3$ (Figure 5(c)). The total elongation of the sample with an initial length $60 \mathrm{~mm}$ is $\sim 100 \%$, and the distribution of the relative elongation along the length of the film is sinusoidal and varies in the range $\lambda=0 \div 2.76 \div 0$ (Figure $5(\mathrm{~d})$ )

In the process of gradual stretching of polymeric composites simultaneously with the elongation of the polymer matrix, redistribution of concentration of functional filler occurs - the volume fraction of functional filler decreases in the direction of propagation of the extension front. The intensity of redistribution of concentration is proportional to the value of relative elongation in a given region of the sample. The features of distribution of concentration of functional filler in local areas directly affect the properties of these areas [37-40].

Graded stretching method can be considered as a scientific and technological innovation for the creation of a new type of FGMs on the base of liner polymers/copolymers and their composites.

FGMs are composites or single-phase materials [42-46], whose properties change continuously or stepwise in at least one direction of particle, film or bulk sample. The change of 
characteristics in FGMs is associated with a corresponding variation of the chemical composition or physical structure of the material.

The unique characteristics of FGMs attract great attention in the scientific community. It is considered that this new concept of designing of material microstructures will revolutionize the field of materials science, as it allows for the first time full integration of material and structural factors in the final design of structural componentry.

In accordance with [43], four main types of microstructure gradation exist-gradation in volume fraction, form, orientation, and size of functional filler. In both natural and synthetic FGMs, more than one type of gradation may be existing simultaneously.

Based on our results, the important conclusion can be made: in addition to the four types of gradation, there is a fifth type of microstructure gradation inherent in thermoplastic polymers-gradient of preferential arrangement of structural elements (macromolecular chains and their parts, as well as supermolecular structural fragments) in stretching direction. The transformation scheme of statistical coils in the process of gradient stretching was shown in Figure 1.

The creation of a new type of microgradation actually means the emergence of new opportunities for research.

Gradient-oriented stretching methods give the possibility to create FGMs by using both known technological approaches-creation of gradient of microstructure or gradient of chemical composition.

In our case, gradient of microstructure corresponds to the gradient of preferential location of structural elements (macromolecular chains, its segments and supermolecular structural fragments) in the stretching direction which is characterized quantitatively by the gradient of orientation/stretching degree. Gradient of microstructure causes the gradient of optical, mechanical, thermal, acoustic, sorption, etc. properties of polymer material. Gradient of microstructure can be formed on the base of single polymer/copolymer. For illustration, we can call for GB (Gradient of Birefringence)-elements, which were obtained by gradient orientation of polyvinyl alcohol and polyethylene terephthalate-films [22-27, 36].

Gradient of chemical composition is created by stretching of polymer composites (in which thermoplastic polymer is a matrix for metal, ceramics, another polymers or pores) in graded mode. As it was shown above in polymer composites containing conductive particles, an increase in the drawing degree leads to a gradual increase in the electrical resistance of composite, which is caused by a gradual reduction of volume concentration of conductive particles in the drawing direction. A similar reasoning is also valid for composites containing a magnetic filler [37-40].

\section{Conclusions}

An algorithm and a mathematical model are proposed for managing the process of forming materials of a new type-gradually and homogeneously oriented linear polymers (polymer composites) with a given distribution of relative elongation. The transformation of isotropic poly- mers (polymer composites) into a gradually or homogeneously oriented state is carried out on a specially designed device for uniaxial, zonal stretching. The design feature of the device is the use of an active cooler with the ability to move adjustable speed/acceleration and direction of movement in the process of stretching the test sample. This provides a predetermined (constant or changing) extent of the stretching zone in the process of drawing the sample. The description and principles of operation of the uniaxial, zonal stretching device are given. The experimental data illustrating the capabilities of the method and the effectiveness of the mathematical modeling are presented. Gradient orientation can be considered as an effective scientific and technical innovation for creating functionally graded materials with a gradient of microstructure (gradient degree of orientation/relative elongation of the polymer matrix) or chemical composition (volume fraction of functional fillers), respectively, based on a single polymer/copolymer or polymer composites containing functional fillers.

\section{Data Availability}

The data used to support the findings of this study are included within the article.

\section{Conflicts of Interest}

The authors declare no conflict of interest.

\section{Acknowledgments}

This research was performed as part of the employment of the authors at the Vl. Chavchanidze Institute of Cybernetics of the Georgian Technical University, supported by the Ministry of Education, Science, Culture and Sport, Georgia.

\section{References}

[1] I. M. Ward, Structure and Properties of Oriented Polymers, Springer Science \& Business Media, London, 2013.

[2] S. Fakirov, Oriented Polymer Materials, Willey-VCH Verlag $\mathrm{GmbH} \& \mathrm{Co} . \mathrm{KGaA}, 2008$.

[3] I. Slutsker, "Oriented state," in Encyclopedia of Polymers, V. Kabanov, Ed., vol. 2, pp. 515-552, Soviet Encyclopedia, Moscow, 1974.

[4] A. Ciferri and I. M. Ward, Ultra High Modulus Polymers, Applied Science Publications, London, 1979.

[5] A. Peterlin, "Drawing and extrusion of semi-crystalline polymers," Colloid and Polymer Science, vol. 265, no. 5, pp. 357382, 1987.

[6] V. Beloshenko, Y. Beygelzimer, and Y. Voznyak, "Solid-state extrusion," in Encyclopedia of Polymer Science and Technology, pp. 1-16, Willey Online Library, 2015.

[7] A. E. Zachariades, T. Kanamoto, and R. S. Porter, "Solid-State coextrusion of high-density polyethylene. II. Effect of molecular weight and molecular weight distribution," Journal of Polymer Science: Polymer Physics Edition, vol. 18, no. 3, pp. 575-585, 1980. 
[8] J. Petermann and R. M. Gohil, "A new method for the preparation of high modulus thermoplastic films," Journal of Materials Science, vol. 14, no. 9, pp. 2260-2264, 1979.

[9] H. Brody, "The extensibility of polyethylene terephthalate fibers spun at high wind-up speeds," Journal of macromolecular Science, Part B, Physics, vol. 22, no. 1, pp. 19-41, 1983.

[10] T. Kunugi, S. Oomori, and S. Mikami, "Preparation of ultrahigh modulus polyethylene films by the zone-annealing method," Polymer, vol. 29, no. 5, pp. 814-820, 1988.

[11] K. Hyungsup, S. C. Kwang, and J. Byung-Chul, "Mathematical modeling of zone drawing process," Macromolecular Research, vol. 12, no. 2, pp. 206-212, 2004.

[12] A. Suzuki and T. Okano, "Zone drawing and zone annealing of poly(ethylene terephthalate) microfiber prepared by $\mathrm{CO}_{2}$ laser thinning," Journal of Applied Polymer Science, vol. 92, pp. 2989-2994, 2004.

[13] V. Tsvetkov, "Birefringence in polymers," in Encyclopedia of Polymers, V. Kargin, Ed., vol. 1, pp. 669-673, Soviet Encyclopedia, Moscow, 1974.

[14] M. Shen and B. Bever, "Gradients in polymeric materials," Journal of Materials Science, vol. 7, no. 7, pp. 741-746, 1972.

[15] L. Nadareishvili, R. Bakuradze, N. Topuridze, L. Sharashidze, and I. Phavlenishvili, "Gradually oriented state of the linear polymers. Section 1. Applications of polymer chemistry and promising technologies. Ch.12," in High-Performance Polymers for Engineering-Based Composites, O. Mukbaniani, M. Abadi, and T. Tatrishvili, Eds., pp. 137-144, Apple Academic Press, Inc., 2015.

[16] L. Nadareishvili, V. Akhobadze, and S. Gvatua, "Device for stretching of polymer films," Georgian Patent P 2992, 2001.

[17] L. Nadareishvili, Z. Wardosanidze, and G. Chelidze, "The method of deformation of polymer films," Georgian Patent $\mathrm{P}$ 4182, 2005.

[18] L. Nadareishvili, R. Bakuradze, N. Topuridze, L. Sharashidze, and I. Phavlenishvili, "Method of obtaining of gradually oriented polymer films. Section 1. Applications of polymer chemistry and promising technologies. Ch. 13," in HighPerformance Polymers for Engineering-Based Composites, O. Mukbaniani, M. Abadie, and T. Tatrishvili, Eds., pp. 145-152, Apple Academic Press, Inc., 2015.

[19] L. Nadareishvili, Z. Wardosanidze, I. Skhirtladze et al., "Device for stretching of polymer films," Georgian Patent P 4398, 2006.

[20] L. Nadareishvili, "Nadareishvili's device for stretching of polymer samples," Georgian Patent P 6509, 2014.

[21] L. Nadareishvili, "Nadareishvili's device for graded or homogeneous stretching of polymer films," Georgian Patent P 6842 B, 2018.

[22] L. Nadareishvili, "Fabrication method and investigation of polymer films with a specified gradient of birefringence," Georgian Engineering News, vol. 2, pp. 73-77, 2001.

[23] N. Lekishvili, L. Nadareishvili, G. Zaikov, and L. Khananashvili, "New Concepts in Polymer Science," in Polymers and Polymeric Materials for Fiber and Gradient Optics, J. S. Vygodsky and S. A. Samsonia, Eds., Utrecht, Boston, Koln, Tokyo, VSP, 2002.

[24] L. Nadareishvili, S. Gvatua, Y. Blagidze, and G. Zaikov, "Gradient of birefringence: A new direction for gradient optics," Journal of Applied Polymer Science, vol. 91, no. 1, pp. 489-493, 2004.

[25] N. Lekishvili, L. Nadareishvili, and G. Zaikov, "Polymer medium with gradient of the optical properties -advanced materials for next-generation optical instrument," in Panorama of modern chemistry of Russia. Advances in the field of physical chemistry of polymers, Chemistry, G. E. Zaikov, Ed., , pp. 624-675, Moscow, 2004.

[26] L. Nadareishvili, N. Lekishvili, and G. E. Zaikov, "Polymer medias with gradient of the optical properties," in Modern Advanced in Organic and Inorganic Chemistry, G. E. Zaikov, Ed., pp. 31-134, Nova Science Publishers, New York, NY, USA, 2005.

[27] N. Lekishvili, L. Nadareishvili, and G. Zaikov, "Polymer materials with the structural inhomogeneities for modern optical devices. (Review)," in New Trends in Natural and Synthetic Polymer Science, G. Vasile and G. Zaikov, Eds., pp. 165-201, Nova Science Publishers, Inc., New York, NY, USA, 2006.

[28] L. Nadareishvili, Z. Wardosanidze, N. Lekishvili, N. Topuridze, G. Zaikov, and R. Kozlovski, "Gradient-Oriented state of polymers: formation and Investigation," Molecular Crystals and Liquid Crystals, vol. 556, no. 1, pp. 52-60, 2012.

[29] L. Nadareishvili, R. Bakuradze, B. Kilosanidze, N. Topuridze, L. Sharashidze, and I. Pavlenishvili, "Graded orientation of the linear polymers," World Academy of Science, Engineering and TechnologyInternational Journal of Chemical and Molecular Engineering, vol. 9, pp. 251-256, 2015.

[30] L. Holliday and I. M. Ward, "Ch. 1. The general introduction to the structure and properties of oriented polymers," in Structure and Properties of Oriented Polymers, Materials Science Series, I. M. Ward, Ed., pp. 22-27, Publisher Springer Science \& Business Media, London, 2013.

[31] L. A. Laius and E. V. Kouvshinsky, "Strength properties of oriented amorphous linear polymers in relation to their structure," Mechanics of Polymers, vol. 3, no. 3, pp. 304307, 1967.

[32] L. Laius and K. Ye, "The influence of molecular weight on strain-strength properties of oriented amorphous polymers," Mechanics of polymers, vol. 4, pp. 579-585, 1967.

[33] N. Shishkin, M. Milagin, and A. Gabarayeva, "Tensile strength of oriented solid amorphous polymers," Mechanics of polymers, vol. 6, pp. 1048-1053, 1967.

[34] S. Frenkel, "Macromolecule, Encyclopedia of Polymers," in Great Soviet Encyclopedia, v. 2, V. Kabanov, Ed., p. 134, Soviet Encyclopedia, Moscow, Russian, 1974.

[35] C. E. Carraher Jr., Seymour/Carraher's Polymer Chemistry, Marcel Dekker, Inc., New-York-Basel, 6th edition, 2005, eLibrary.

[36] B. Kilosanidze, G. Kakauridze, L. Nadareishvili, and Y. Mshvenieradze, "New method for determining the distribution of birefringence and linear dichroism in polymer materials based on polarization-holographic grating," World Academy of Science, Engineering and Technology, International Journal of Chemical and Molecular Engineering, vol. 9, pp. 1340-1344, 2015.

[37] J. Aneli, L. Nadareishvili, G. Mamniashvili, A. Akhalkatsi, and G. Zaikov, "Gradiently anisitropic conducting and magnetic polymer composites," Chemistry \& Chemical Technology, vol. 6, pp. 285-289, 2012.

[38] J. Aneli, L. Nadareishvili, A. Akhalkatsi, M. Bolotashvili, and G. Basilaia, "Polymer composites with gradient of electric and magnetic properties," Problems of Mechanics, vol. 4, pp. $78-84,2014$. 
[39] J. Aneli, L. Nadareishvili, and L. Shamanauri, "Electric and magnetic properties of graded oriented polymer composites," Journal of Electrical Engineering, vol. 4, pp. 196-202, 2016.

[40] J. Aneli, L. Nadareishvili, G. Mamniashvili, A. Akhalkatsi, and G. Zaikov, "Section III: a note on gradiently anisotropic conducting and magnetic polymer composites," in Polymer Products and Chemical Processes. Techniques, Analysis, and Applications, R. A. Pethrick, E. M. Pearce, and G. E. Zaikov, Eds., pp. 101-124, Apple Academic Press, 2013.

[41] C. M. Costa, J. Gomes, J. SerradoNunes, S. Sencadas, and S. Lanceros-Mendes, "Functionally graded electroactive poly(vinylidene fluoride) polymers," International Journal of Materials and Product Technology, vol. 39, no. 1-2, pp. 178-185, 2010.

[42] Y. Miyamoto and I. Shiota, Functionally Graded Materials, Elsevier Science, New-York, NY, USA, 1996.

[43] A. Neubrand, "Functionally graded materials," in Encyclopedia of Materials: Science and Technology (Second Edition), K. H. J. Buschow, W. C. Robert, C. F. Merton, I. Bernard, J. K. Edward, M. Subhash, and V. Patrick, Eds., pp. 3407-3413, Elsevier, New-York (NY): Oxford, 2001.

[44] B. Kieback, A. Neubrand, and H. Riedel, "Processing techniques for functionally graded materials," Materials Science and Engineering: A, vol. 362, no. 1-2, pp. 81-106, 2003.

[45] N. Reinolds, Functionally Graded Materials, Nova Science Publication, New-York, NY, USA, 2011.

[46] J. Stabik, M. Chomiak, A. Dybowska et al., "Chosen manufacture methods of polymeric graded materials with electrical and magnetic properties gradation," Journal of Achievements in Materials and Manufacturing Engineering, vol. 54, pp. 218226, 2012. 


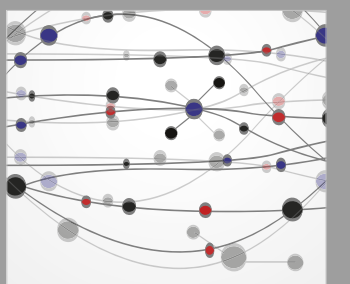

The Scientific World Journal
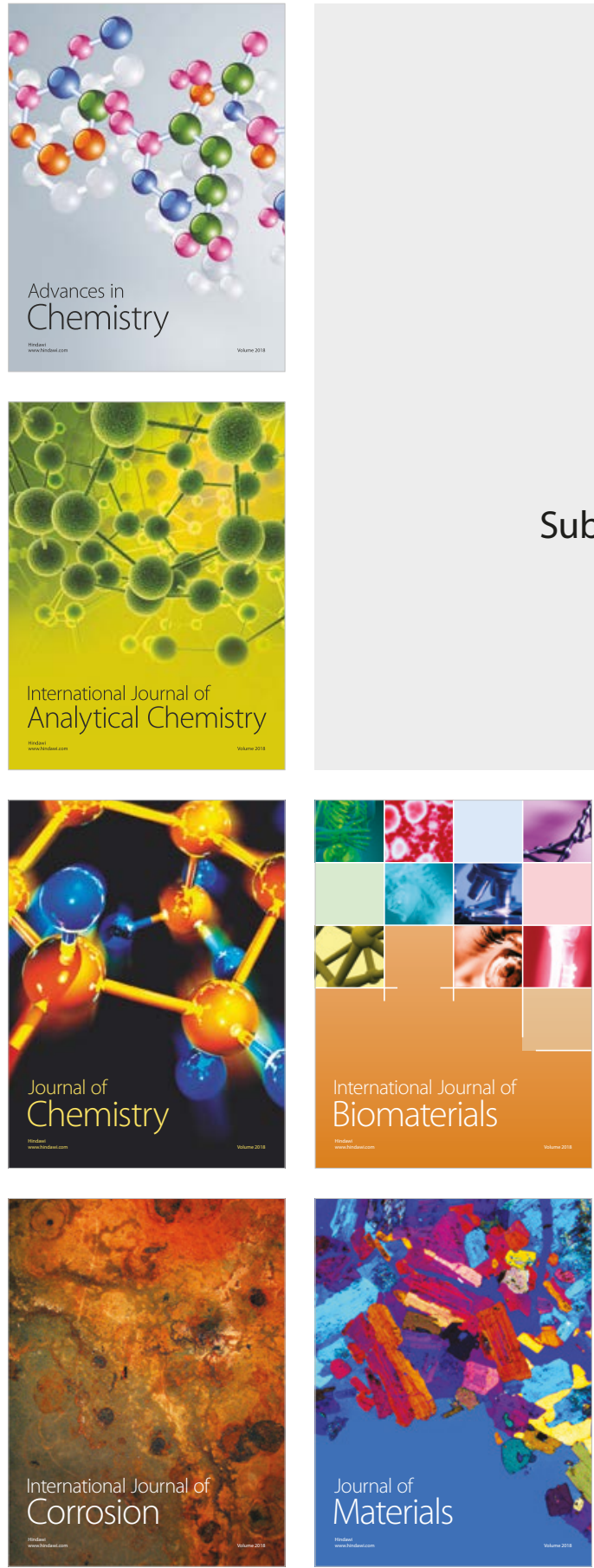

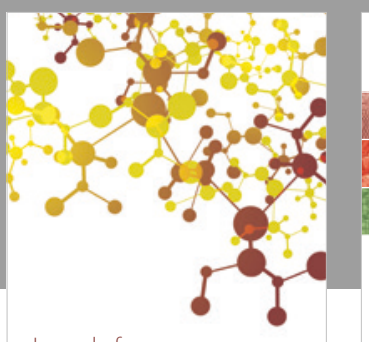

Journal of

Applied Chemistry
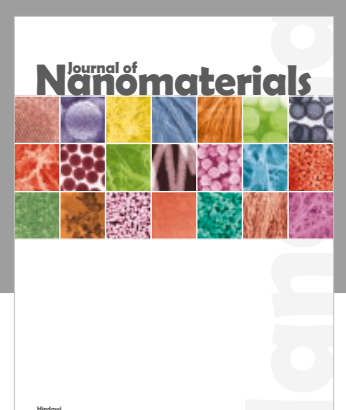

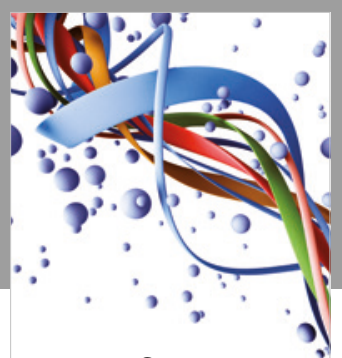

Scientifica

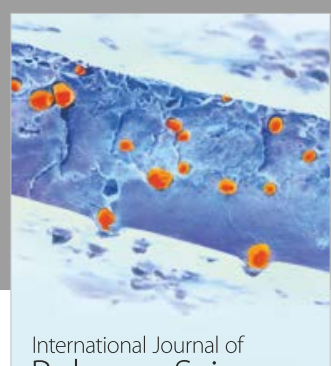

Polymer Science

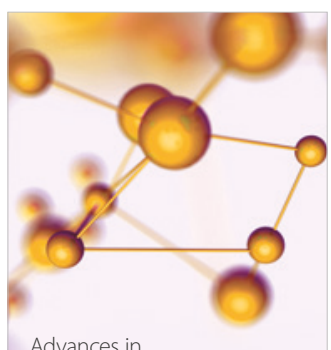

Physical Chemistry
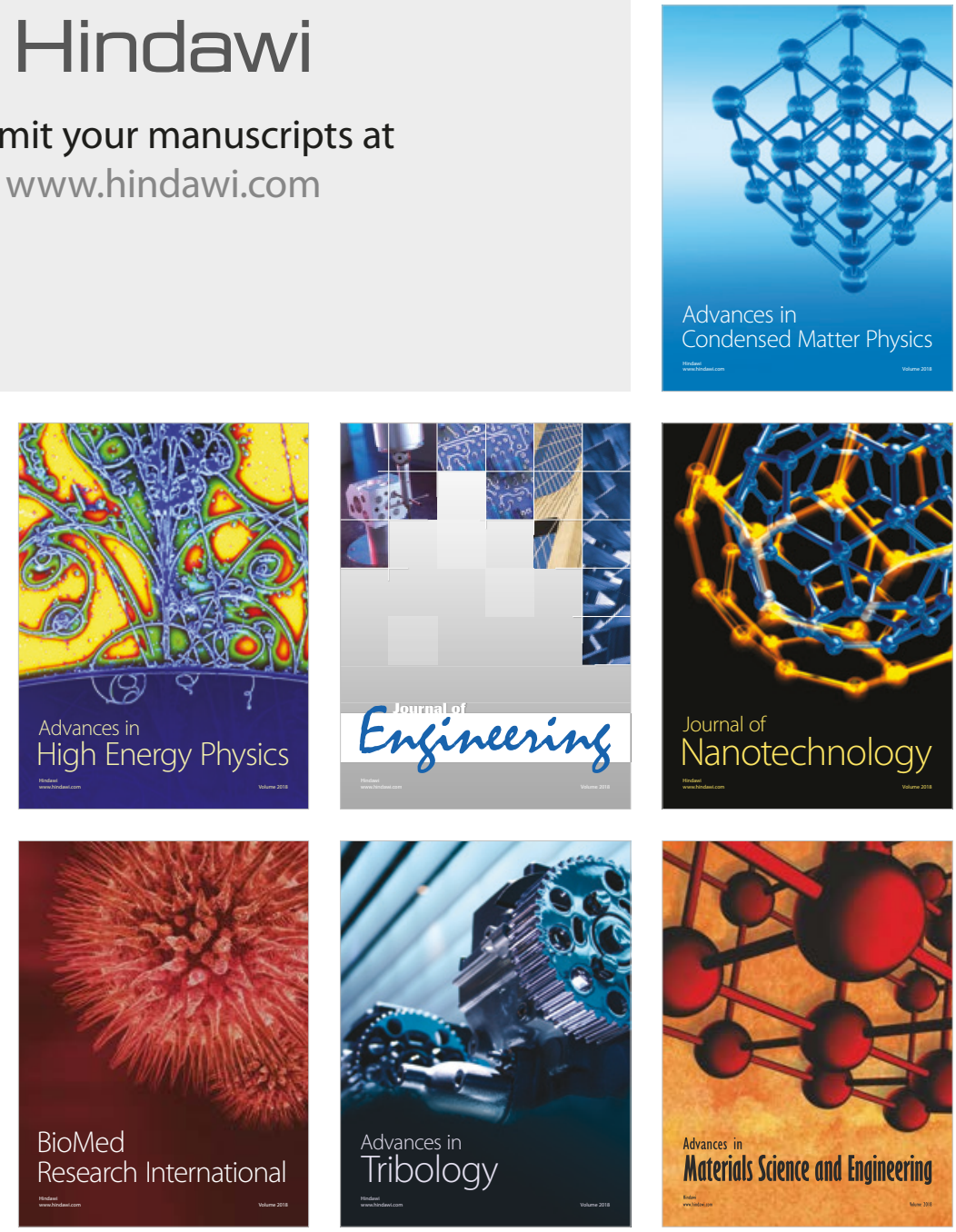